Document downloaded from:

http://hdl.handle.net/10251/146181

This paper must be cited as:

Cuenca, A.; Zheng, M.; Tomizuka, M.; Sanchez, S. (08-2). Non-uniform Multi-rate Estimator based Periodic Event-Triggered Control for resource saving. Information Sciences. 459:86102. https://doi.org/10.1016/J.INS.2018.05.038

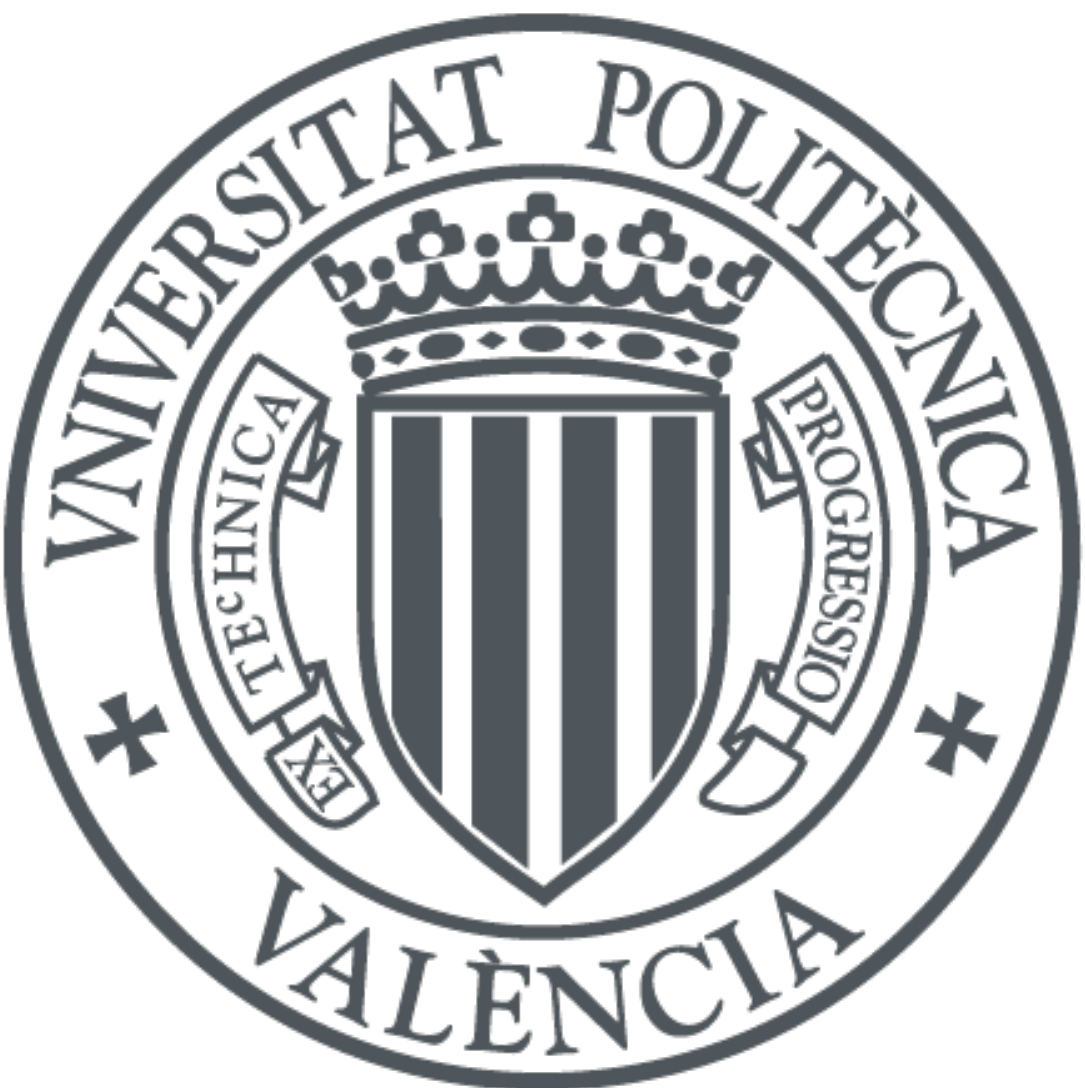

The final publication is available at

https://doi.org/10.1016/J.INS.2018.05.038

Copyright Elsevier

Additional Information 


\title{
Non-uniform Multi-rate Estimator Based Periodic Event-Triggered Control for Resource Saving ${ }^{\text {th }}$
}

\author{
Ángel Cuenca, Minghui Zheng, Masayoshi Tomizuka, Sergio Sánchez
}

\begin{abstract}
This paper proposes a systematic non-uniform multi-rate estimation and control framework for a periodic event-triggered system which is subject to external disturbance and sensor noise. When the disturbance dynamic model is available, and in order to efficiently estimate the state variable and disturbance from non-uniform slow-rate measurements, a time-varying Kalman filter is designed. When the disturbance dynamic model is not available, a disturbance observer is proposed as an alternative approach. Both the Kalman filter and the disturbance observer are proposed in a non-uniform multi-rate format. Such disturbance estimation enables faster controller updating in spite of slower measurement. Interlacing techniques are used in the control system to uniformly distribute the computational load at each fast sampling instance. Compared to the conventional time-triggered sampling paradigm, the control solution is able to reduce the resource utilization, while maintaining a satisfactory control performance. The proposed control solution will reduce the number of transmissions among devices, which enhances the energy and computational efficiency. Simulation results are provided to validate the effectiveness and benefits of the proposed control algorithms.
\end{abstract}

\footnotetext{
"Original Article Statement: a) This manuscript is the authors' original work and has not been published nor has it been submitted simultaneously elsewhere. b) All authors have checked the manuscript and have agreed to the submission. The corresponding author is A. Cuenca. He is with Departamento de Ingeniería de Sistemas y Automática (DISA), Instituto Universitario de Automática e Informática Industrial (AI2), Universitat Politècnica de València (UPV), and he was a Visiting Fulbright Scholar at the Mechanical Systems Control (MSC) Laboratory, Mechanical Engineering (ME) Department, University of California, Berkeley (UC Berkeley) (e-mail: acuenca@isa.upv.es); M. Zheng and M. Tomizuka are with the MSC Lab, ME Dept., UC Berkeley (e-mails: minghuizheng@berkeley.edu, tomizuka@berkeley.edu); and S. Sánchez is with DISA, UPV (e-mail: sersncca@etsid.upv.es).
} 
Keywords: Event-Triggered Sampling, Kalman Filter, Multi-rate Control, Disturbance Observer.

Table 1: Abbrevations

\begin{tabular}{|l|l|}
\hline ETS & Event-Triggered Sampling \\
\hline TTS & Time-Triggered Sampling \\
\hline PETC & Periodic Event-Triggered Control \\
\hline MRIC & Multi-Rate Controller with Interlacing \\
\hline NUMRE & Non-Uniform Multi-Rate Estimator \\
\hline DOB & Disturbance Observer \\
\hline WSN & Wireless Sensor Networks \\
\hline NCS & Networked Control System \\
\hline HDD & Hard Disk Drive \\
\hline ZOH & Zero-Order Hold \\
\hline MTM & Mixed Triggered Mechanism \\
\hline ESO & Extended State Observer \\
\hline LFT & Linear Fractional Transformation \\
\hline$C S$ & Computation Saving \\
\hline$I S E$ & Integral Squared Error \\
\hline$I S E P$ & Integral Squared Error for PETC \\
\hline$N_{E}$ & Sampling efficiency \\
\hline$U_{c}, U_{a}$ & Utilization of the controller, and actuator \\
\hline$N_{s c}^{\mathrm{ETS}}$, & $\begin{array}{l}\text { Number of sensor-to-controller, } \\
\text { controller-to-actuator transmissions } \\
N_{c a}^{\mathrm{ETS}}\end{array}$ \\
\hline$N^{\mathrm{TTS}}$ & the ETS \\
\hline$C_{c}$ & Number of transmissions for the TTS \\
\hline$G P I$ & Amount of computation by the controller \\
\hline
\end{tabular}

\section{Introduction}

Event-Triggered Sampling (ETS) in control systems has been initialized in the past decades [17] and it has been become a trending research direction recently $[36,25,16,10]$ because of its wide applications in emerging areas such as Wireless Sensor Networks (WSN), and Networked Control Systems 
(NCS) where similar problems like network-induced delays [7, 9, 43, 40], packet dropouts [7, 27,39], and packet disorder [6, 28, 46] are faced as well.

In the Time-Triggered Sampling (TTS) strategy the plant is periodically sampled, while in the ETS strategy the plant is sampled only when the norm of the output errors exceeds certain thresholds, which is considered as an event. The integration of TTS and ETS paradigms results in a Periodic Event-Triggered Control (PETC) technique [16, 45, 44, 14, 3], in which the event-triggering conditions are evaluated periodically. The PETC scenario can be developed in both continuous-time (see [18], and references therein) and discrete-time frameworks (see [25] among others). In this paper, the PETC is incorporated with a Multi-Rate Controller with Interlacing (MRCI) along with a Non-Uniform Multi-Rate Estimator (NUMRE) and a Disturbance Observer (DOB), when it is required. Compared to the TTS, ETS enables to can further reduce the resource utilization (mainly, data transfer, processing power, and energy consumption). This paper exploits PETC to reduce the number of transmissions. It means that only when the eventtriggered conditions that are evaluated periodically hold, the packets are transmitted. This would reduce the utilization of the control system devices, and result in considerable reductions in the overall energy usage. For example, in wireless networks, the network devices are battery-powered, and the reductions would extend the lives of the batteries in the devices [16, 29]. Since the ETS strategy utilizes less system information, the control performance may be worsened [42] compared to the desired one defined by the TTS case. One solution to solve this problem is to include the controller in the eventtriggering decision process (see $[36,25]$ and references therein). However, it may compromise the resource saving. Furthermore, event-based sampling and control heavily depend on each other, and changing one requires a redesign of the other to guarantee the stability and desired performance for the closed-loop system. A more versatile option is to decouple the event-based sampling and control by including event-based estimation techniques. Without these techniques, relevant system information on inter-sampling times would not be available. With such inter-sampling information, both resource saving and performance criteria can be guaranteed.

There are some existing literature that addresses various challenges on the event-based state estimation problem. In [16], a Luenberger observer at the sensor device and a model-based predictor, that runs both at the sensor and the controller, were proposed to estimate the state of the plant. In [11], a nominal model of the system, which was incorporated into the ac- 
tuator/controller node in order to estimate the state between non-periodic updating intervals with time-varying delays, is presented. In [19], an $H_{\infty}$ filter for NCS with communication delay is developed. In $[12,15]$, distributed event-triggered filters over Sensor Networks (SN) by employing $H_{\infty}$ control techniques are designed. In [24], distributed event-triggered filtering over WSN is proposed using a recursive algorithm to obtain an upper bound of the filtering error variance. In [13], event-triggered distributed set-membership estimators are constructed by taking the influence of unknown-but-bounded process and measurement noise into account. In [27, 34], networked estimation problems are presented using modified Kalman filters to deal with packet dropouts. In [32], a Gaussian sum filter is introduced. In [26], a modified Kalman filter with intermittent measurement updates is investigated for a household network. In [37], time-varying Kalman filters are used to estimate the state conditioned on the received measurements in a multi-agent framework.

This paper follows a recent line of research [26, 37], in which the celebrated Kalman filter is considered in a time-varying fashion. The filter includes a slow-rate non-uniform measurement update (due to the eventbased sampling policy) along with a fast-rate model-based prediction, which is a Non-Uniform Multi-Rate Estimator. While in previous works the estimator periodically calculates estimated state even when the sensor nodes do not transmit data, in this paper the state is only updated when receiving a measurement (when the event is triggered), but computing at this moment an $h$-step ahead cascade prediction. Then, the control stage is not needed to work at any synchronous time but only when a measurement is received, resulting in less device usage. To the best of the authors' knowledge, this working mode is novel in this kind of frameworks.

Regarding the cascade prediction approach used in this work, similar ones are utilized by [48] which is in a uniform multi-rate scenario (with a timeinvariant Kalman filter gain), [16] which is based on a simple model-based predictor, and $[42,41]$ which is exploiting model-based predictions to compensate for network-induced delays. Kalman filtering can be indistinctly used for the scenario where non-delivery of measurement data results from packet drops or from an event-based mechanism. The prediction and estimation capabilities of the proposed Kalman filter are able to address typical problems in Networked Control Systems (NCS) such as network-induced delays and packet dropouts. To be more specific, it is relevant for instance in networkbased rehabilitation systems [1], which are based on battery-powered wireless 
devices and limited computing power microprocessors, and human-machine systems [23], which requires both safety and efficient interactions among human workers and robots.

In this work, the plant is subject to some external disturbance. If the disturbance dynamics is known, the disturbance is estimated from the state estimates (computed by the Non-Uniform Multi-Rate Estimator). When the

dynamics is not available, the disturbance estimation is carried out by adding a Disturbance Observer to the control system [22, 49]. The controller is designed as a Multi-Rate Controller with Interlacing $[8,30]$ to compensate the disturbance and to guarantee closed-loop performance. Besides the benefits of computation saving in Periodic-Event Triggered Control solution, the consideration of the interlacing technique enables additional saving of the computation resources by uniformly distributing them at each fast sampling instance.

The main contribution of this paper is the development of a new and comprehensive approach, where a Non-Uniform Multi-Rate Estimator (NUMRE), a Multi-Rate Controller with Interlacing (MRCI), and optionally a Disturbance Observer (DOB) are systematically brought together in a Periodic Event-Triggered Control (PETC) framework in order to significantly reduce resource utilization, while maintaining a satisfactory control performance.

The remainder of the paper is organized as follows. Section 2 describes the event-triggered control system. Section 3 presents different control structures and event-based conditions included in the control system. Section 4 analyzes the trade-off between control performance and resource utilization, and how to find optimal threshold values for the event-based conditions. In section 5 , a numerical example coming from a practical Hard Disk Drive (HDD) plant model is studied and simulated by means of Truetime [4] (a Matlab/Simulinkbased simulation tool) and the effectiveness of the proposed algorithm is validated. Section 6 concludes the paper.

\section{Problem description}

The control system considered in this paper is depicted in Figure 1. It has two components: the plant side which includes sensor, actuator, and plant (subject to disturbance $d_{k}$ and noise $v_{k}$ signals), and the control side which includes a reference generator, a Multi-Rate Controller with Interlacing (MRCI), a Non-Uniform Multi-Rate Estimator (NUMRE), and an optional Disturbance Observer (DOB). Two different Event-Triggered (ET) 


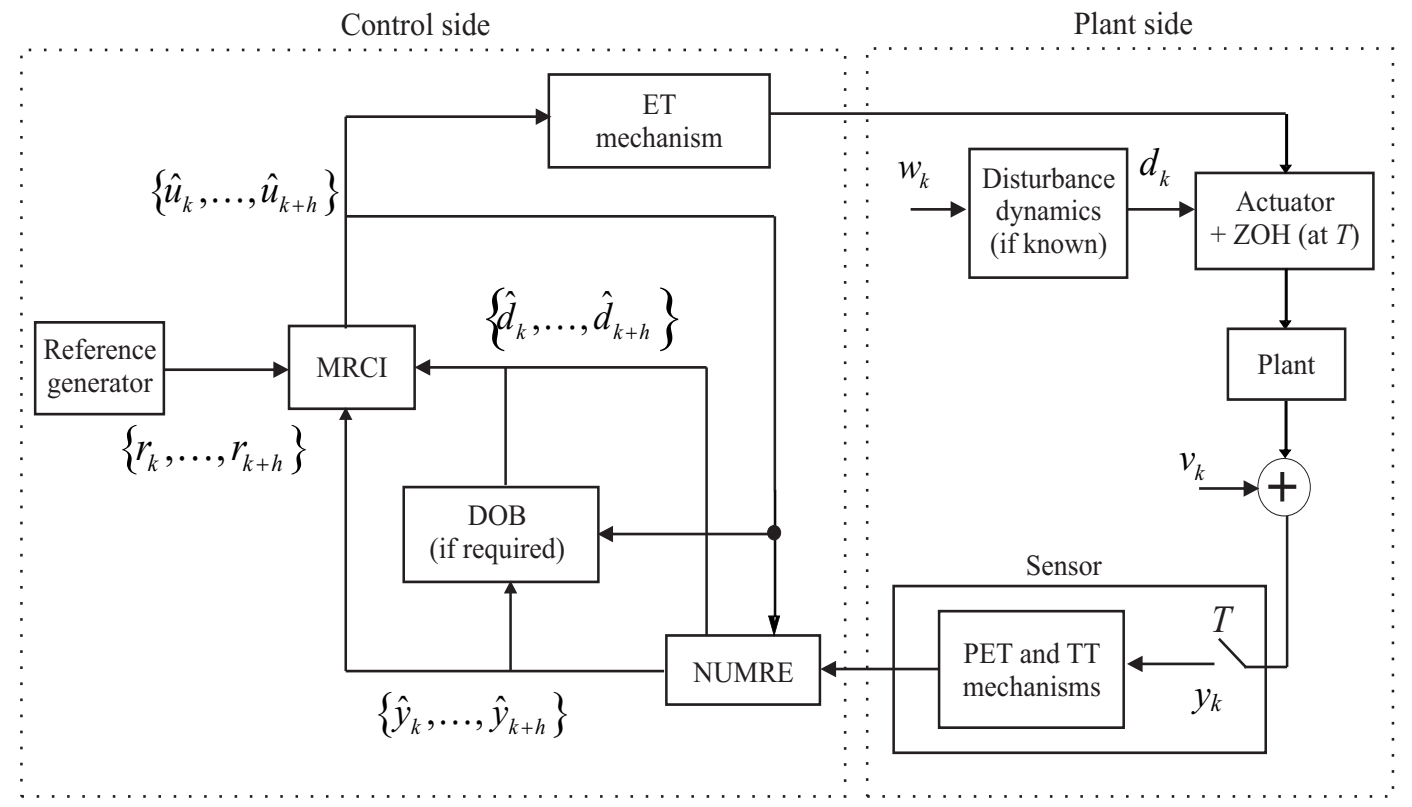

Figure 1: Control system

conditions will be evaluated in the control system. One is related to the system output (defined at the sensor device), and the other one is related to the control action (defined at the control side). The main difference between the two ET conditions is: whereas at the sensor device the condition is periodically evaluated (Periodic ET -PET- condition), at the controller side the condition is evaluated only when a measurement is received from the sensor (ET condition). In addition, a Time-Triggered (TT) condition will be included at the sensor for safety reasons or reference tracking and to ensure that a new, updated process output measurement is put into the control system after elapsing the maximum predefined time.

Let us denote $\hat{x}_{(i \mid j)}$ as the estimate of the state $x_{i}$ based on the measurement up to time instant $j$, where $j \leq i$. The proposed framework works as follows (more details are in Section 3):

- When the Periodic Event-Triggered or the Time-Triggered condition holds at the sensor device, a new output $y_{k}(k \in \mathbb{N}$ are the time index with period $T$ ) is received at the control side. Then, the control side calculates the amount of $T$-periods elapsed between the previous and current output. This value is known as multiplicity $N$, which is time- 
varying due to the non-uniform nature of the output pattern that is received by the control side.

- From $N$, the gain $K(N)$ of the Non-Uniform Multi-Rate Estimator (Kalman filer) (NUMRE) will be computed and the correction of the estimator will be carried out, yielding the estimate of the state, $\hat{x}_{(k \mid k)}$.

- From $\hat{x}_{(k \mid k)}$, the estimated (corrected, filtered) output $\hat{y}_{k}$ and the estimated disturbance $\hat{d}_{k}$ (if the disturbance dynamics are known) can be obtained. When the disturbance model is not provided, a Disturbance Observer can be added to get $\hat{d}_{k}$.

- From $\hat{y}_{k}, \hat{d}_{k}$, and the reference $r_{k}$, the Multi-Rate Controller with Interlacing (MRCI) is used to compute the current (estimated) control action $\hat{u}_{k}$ in order to reach the desired control performance by compensating for the actual disturbance $d_{k}$.

- From $\hat{u}_{k}$ and the plant model, the NUMRE's prediction stage can be performed, yielding $\hat{x}_{(k+1 \mid k)}$. Then, $\hat{y}_{k+1}$ and $\hat{d}_{k+1}$ can be calculated, and hence, the MRCI is able to compute the next $\hat{u}_{k+1}$ (taking into account $r_{k+1}$ as well). Following this cascade prediction procedure up to $h$-step ahead estimations, $\left\{\hat{u}_{k+1}, \ldots, \hat{u}_{k+h}\right\}$ can be obtained.

- If the Event-Triggered condition at the control side holds, the current control action $\hat{u}_{k}$ and the $h$ future ones $\left\{\hat{u}_{k+1}, \ldots, \hat{u}_{k+h}\right\}$ are sent to the actuator in a packet. Therefore, the actuator injects (via Zero-Order Hold -ZOH-) the current control action $\hat{u}_{k}$ at instant $k$, and the future ones $\left\{\hat{u}_{k+1}, \ldots, \hat{u}_{k+h}\right\}$ at instants $k+1, \ldots, k+h$ while no new control action is received for the $h$ future instants.

\section{Control solution}

The following subsections provide detailed definition for each element in the control systems.

\subsection{Plant side}

\subsubsection{Plant model}

The plant model with sampling period $T$ is

$$
\begin{aligned}
x_{k+1}^{p} & =A_{p} x_{k}^{p}+B_{p} \hat{u}_{k}+B_{p} d_{k} \\
y_{k} & =C_{p} x_{k}^{p}+v_{k}
\end{aligned}
$$


where $y_{k}$ is the measurement, $\hat{u}_{k}$ is the control signal, $d_{k}$ is the disturbance signal, and $v_{k}$ is the measurement noise. $x_{k}^{p}$ is the plant state variable, and $A_{p}, B_{p}, C_{p}$ are matrices with proper dimensions.

Assume the disturbance $d_{k}$ is generated through the following dynamics (driven by broadband noise $w_{k}$ ),

$$
\begin{aligned}
x_{k+1}^{d} & =A_{d} x_{k}^{d}+B_{d} w_{k} \\
d_{k} & =C_{d} x_{k}^{d}
\end{aligned}
$$

where $x_{k}^{d}$ is the disturbance state, and $A_{d}, B_{d}, C_{d}$ are matrices with suitable dimensions. Then the system is augmented as follows,

$$
\begin{aligned}
{\left[\begin{array}{c}
x_{k+1}^{p} \\
x_{k+1}^{d}
\end{array}\right] } & =\left[\begin{array}{cc}
A_{p} & B_{p} C_{d} \\
0 & A_{d}
\end{array}\right]\left[\begin{array}{c}
x_{k}^{p} \\
x_{k}^{d}
\end{array}\right]+\left[\begin{array}{c}
B_{p} \\
0
\end{array}\right] \hat{u}_{k}+\left[\begin{array}{c}
0 \\
B_{d}
\end{array}\right] w_{k} \\
y_{k} & =\left[\begin{array}{ll}
C_{p} & 0
\end{array}\right]\left[\begin{array}{l}
x_{k}^{p} \\
x_{k}^{d}
\end{array}\right]+v_{k} \\
d_{k} & =\left[\begin{array}{ll}
0 & C_{d}
\end{array}\right]\left[\begin{array}{l}
x_{k}^{p} \\
x_{k}^{d}
\end{array}\right]
\end{aligned}
$$

where

$$
\begin{aligned}
& A=\left[\begin{array}{cc}
A_{p} & B_{p} C_{d} \\
0 & A_{d}
\end{array}\right], B=\left[\begin{array}{c}
B_{p} \\
0
\end{array}\right], B_{w}=\left[\begin{array}{c}
0 \\
B_{d}
\end{array}\right] \\
& C=\left[\begin{array}{ll}
C_{p} & 0
\end{array}\right], \bar{C}=\left[\begin{array}{ll}
0 & C_{d}
\end{array}\right], x=\left[\begin{array}{l}
x_{k}^{p} \\
x_{k}^{d}
\end{array}\right]
\end{aligned}
$$

It is further written in the following compact format

$$
\begin{aligned}
x_{k+1} & =A x_{k}+B \hat{u}_{k}+B w_{w} \\
y_{k} & =C x_{k}+v_{k} \\
d_{k} & =\bar{C} x_{k}
\end{aligned}
$$

\subsubsection{Periodic Event-Triggered (PET) and Time-Triggered (TT) conditions} at the sensor device

At the sensor device, both PET and TT conditions will be evaluated and the sensor is configured as a mixed sampling based device.

Let $\beta_{k} \in\{0,1\}$ denote the scheduling variable at the sensor, where $\beta_{k}=1$ denotes transmission of the sensor data $y_{k}$, and $\beta_{k}=0$ denotes no transmission. The latest sensor data is stored in $\bar{y}_{k}$. Therefore:

$$
\bar{y}_{k}=\beta_{k} y_{k}+\left(1-\beta_{k}\right) \bar{y}_{k-1}
$$


Regarding the Periodic Event-Triggered condition at the sensor, a Mixed Triggered Mechanism (MTM) [2] is implemented based on the system output in such a way that the output is sent to the control side when

$$
\left\|\bar{y}_{k-1}-y_{k}\right\|^{2}>\sigma_{s}\left\|y_{k}\right\|^{2}+\delta_{s}
$$

where $\sigma_{s}$ and $\delta_{s}$ are positive constants. The idea behind this is: when the measurement exceeds the threshold defined by (6), the measurement is sent to the control side to calculate a new control signal; otherwise, the measurement will not be sent in order to save energy and computation.

Regarding the Time-Triggered condition at the sensor, it can be defined from the following two different points of view:

- Safety reasons [31]. In this case, the condition is triggered when a maximum predefined time $t_{\max }=k_{\max } T\left(k_{\max } \in \mathbb{N}\right)$ is elapsed from the moment in which the sensor sent the last measurement $t_{l m}=k_{l m} T$ $\left(k_{l m} \in \mathbb{N}\right)$. Therefore, additionally to (6), the measurement $y_{k}$ is sent to the remote side when

$$
k_{\max }=k-k_{l m}
$$

where $k_{\max }$ can be chosen depending on safety requirements or process dynamics.

- Quick detection of possible changes in the reference signal $r_{k}$. If the reference were predefined, the moments in which this signal changes would be known. In this case, the condition is triggered when the predefined time $t_{\max }$ is elapsed from the last moment in which the reference changed $t_{l r}=k_{l r} T\left(k_{l r} \in \mathbb{N}\right)$. Then, additionally to (6), the measurement $y_{k}$ is sent to the control side when

$$
k_{\max }=k-k_{l r}
$$

\subsection{Control side}

\subsubsection{Non-Uniform Multi-Rate Estimator (NUMRE)}

For single-rate system in (4), conventional Kalman filter provides the best linear estimation for $x_{k}$ in the sense of mean square error when $w_{k}$ and $v_{k}$ are zero-mean white noises [33]. To save the computation cost, the measurement $y_{k}$ is not returned at every sampling instant, which results in a multi-rate 
estimator including predictions and corrections [48]. The prediction is an open-loop estimate process before the next measurement comes; the correction is made to the estimation once the next measurement is available. The combination of prediction with faster updating and estimation with slower correction results in a multi-rate estimator. When the multiplicity $N$ is timevarying, this estimation strategy becomes Non-Uniform Multi-Rate Estimator (NUMRE). We propose a NUMRE in this paper based on the techniques of Extended State Observer (ESO) [47] and multi-rate Kalman filter [48, 35], which allows non-uniform multi-rate estimation for both the state and the disturbances.

According to (6) (and, additionally, to (7) and (8)), the measurements sensed at the fast rate $1 / T$ arrive to the control side at a slower rate and follow non-uniform patterns. Previously, in section 2, the multiplicity $N$ was introduced. Now, it is defined in more detail, from the previous measurement received by the control side $\bar{y}_{k-1}$ (which was sensed in time, say, $k_{l s} T$ ) and the current received measurement $\bar{y}_{k}=y_{k}$. Hence

$$
N=k-k_{l s}
$$

Due to the non-uniform nature of the pattern, $N$ will be time-varying.

From the current received measurement, the NUMRE can compute $h$ step ahead state predictions at the fast rate. As a multi-rate structure, to be defined, a lifted representation [21] for the system in (4) is needed

$$
\begin{aligned}
x_{k+N} & =A^{N} x_{k}+\sum_{c=0}^{N-1} A^{N-1-c} B \hat{u}_{k+c} \\
& +\sum_{c=0}^{N-1} A^{N-1-c} B_{w} w_{k+c} \\
y_{k} & =C x_{k}+v_{k}
\end{aligned}
$$

where the estimator is based on a multi-rate Kalman filter [35, 48], and $w_{k}$, $v_{k}$ are assumed to be zero-mean, white, and Gaussian noises in order to reach the best linear estimation in the sense of mean square error for the augmented state [33].

Taking into account the representation in (10), and remembering that $\hat{x}_{(i \mid j)}$ denotes the estimate of the state $x_{i}$ based on the measurement previously taken at instant $j$, the $h$-step ahead prediction stage and the correction/filtering stage of the NUMRE are designed as follows (and depicted in Figure 2): 
- Correction/filtering stage: at time $k$, there is a new measurement $y_{k}$ which can be utilized to make correction for the prediction $\hat{x}_{(k \mid k)}$,

$$
\begin{aligned}
\hat{x}_{(k \mid k)} & =\hat{x}_{(k \mid k-N)} \\
& +K(N)\left[y_{k}-C \hat{x}_{(k \mid k-N)}\right]
\end{aligned}
$$

where $K(N)$ is dependent on the time-varying $N$ and is obtained from multi-rate Kalman filter,

$$
\begin{aligned}
K(N) & =M_{k+1} C^{\mathrm{T}}\left[C M_{k+1} C^{\mathrm{T}}+V\right]^{-1} \\
M_{k+1} & =A^{N} M_{k}\left(A^{N}\right)^{\mathrm{T}}+W_{e} \\
& -A^{N} M_{k} C^{\mathrm{T}}\left[C M_{k} C^{\mathrm{T}}+V\right]^{-1} C M_{k}\left(A^{N}\right)^{\mathrm{T}}
\end{aligned}
$$

where $V=\operatorname{Cov}(v)$ and

$$
\begin{aligned}
W_{e} & =\operatorname{Cov}\left(\sum_{c=0}^{N-1} A^{N-1-c} B_{w} w_{k+c}\right)=\operatorname{Cov}\left(w_{e}\right) \\
& =E\left\{w_{e} w_{e}^{T}\right\} \\
& =\left(\sum_{c=0}^{N-1} A^{N-1-c} B_{w}\right) W\left(\sum_{c=0}^{N-1} A^{N-1-c} B_{w}\right)^{T}
\end{aligned}
$$

where $W=\operatorname{Cov}(w)$ and $E\{\cdot\}$ denotes the expectation.

- Prediction stage: there is no new measurement available between the previous measurement $y_{k}$ and the next measurement $y_{k+N}$; the best estimates $\hat{x}_{(k+l \mid k)}(l=1, \ldots, h)$ come from the prediction based on the open-loop dynamics,

$$
\hat{x}_{(k+l \mid k)}=A^{l} \hat{x}_{(k \mid k)}+\sum_{c=0}^{l-1} A^{l-1-c} B \hat{u}_{k+c}
$$

where $h$ must be defined in such a way that the maximum value of $N, N_{\max }$, fulfills $h \geq N_{\max }$. In this way, the ahead state $\hat{x}_{(k+N \mid k)}$ required to compute the NUMRE's correction/filtering stage will be available. In addition, from $\hat{x}_{(k+l \mid k)}$, the set of $h$ estimated outputs and disturbances can be calculated:

$$
\begin{aligned}
& \hat{y}_{k+l}=C \hat{x}_{(k+l \mid k)} \\
& \hat{d}_{k+l}=\bar{C} \hat{x}_{(k+l \mid k)}
\end{aligned}
$$




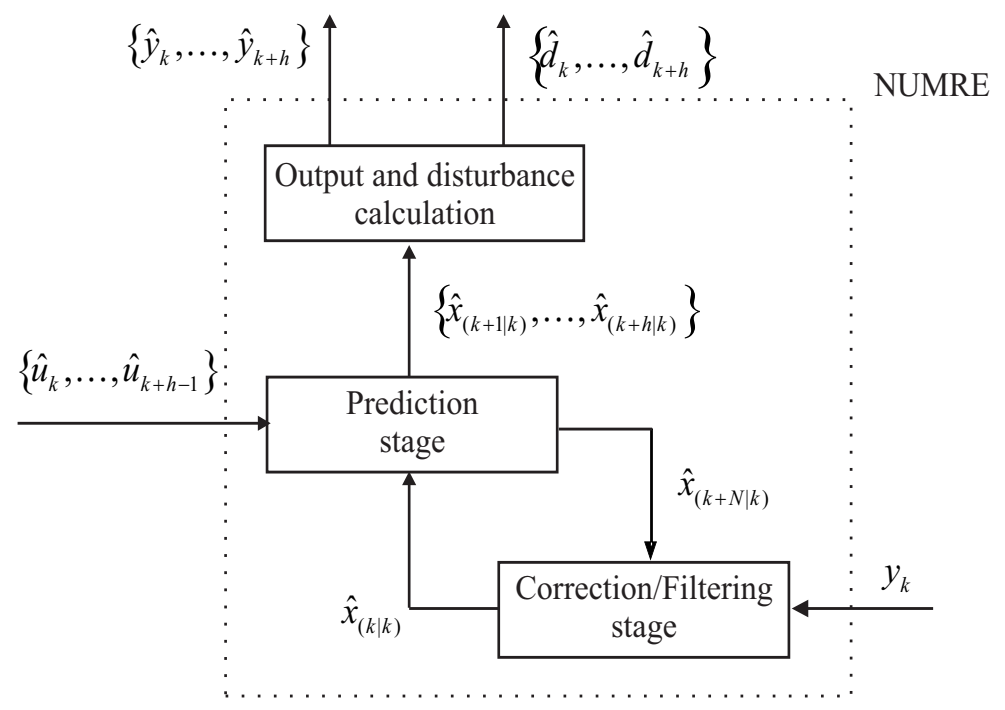

Figure 2: Structure of the Non-Uniform Multi-Rate Estimator

\subsubsection{Disturbance Observer (DOB)}

The estimate of the disturbance $\hat{d}_{k}$ can be obtained from (15), which requires the disturbance dynamic model. When such a model is not available, the disturbance estimation can be carried out by adding a Disturbance Observer (DOB) to the control system. This paper designs the DOB through the optimization method based on $H_{\infty}$ synthesis, which is proposed in [49]. From the transfer function representation, let us denote $G(z)$ as the model of the plant in (1), and $C(z)$ as the baseline feedback controller. The structure of the DOB is shown in Figure 3, where $D(z)=\left[D_{1}(z), D_{2}(z)\right], D_{1}(z)$ is a filter closed to -1 , and $D_{2}(z)$ is a stable casual filter closed to the inverse of $G(z)$. From Figure 3, the disturbance estimation can be obtained from

$$
\hat{d}=D_{1}(z) \hat{u}+D_{2}(z) \hat{y}
$$

where $D(z)$ is obtained through solving the following optimization problem

$$
\begin{aligned}
\min _{D(z), \gamma} \gamma & \\
\text { s.t. } & F_{l}(M(z), D(z)) \text { stable } \\
& \left\|F_{l}(M(z), D(z))\right\|_{\infty}<\gamma
\end{aligned}
$$


In (17), $F_{l}$ stands for the Linear Fractional Transformation (LFT), and

$$
M=\left[\begin{array}{cc}
1 & -1 \\
-C(1+G C)^{-1} G & 1-C(1+G C)^{-1} G \\
(1+G C)^{-1} G & (1+G C)^{-1} G
\end{array}\right]
$$

where $z$ is omitted for simplicity.

Note that, in this case, $\hat{y}_{k}$ is computed by the Non-Uniform Multi-Rate Estimator with no knowledge of the dynamic model of the disturbance, that is, from $\left(A_{p}, B_{p}, C_{p}\right)$ in (1) instead of from the augmented system in (3).

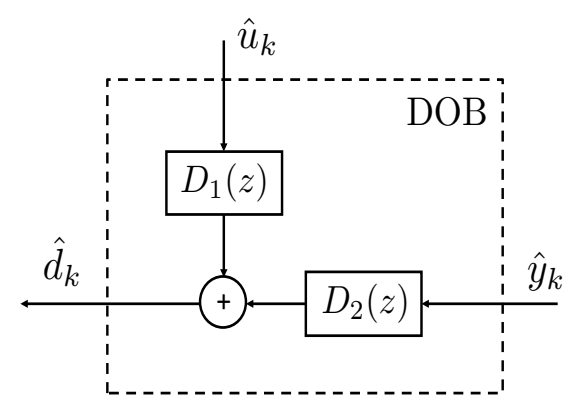

Figure 3: Structure of the Disturbance Observer [49]

\subsubsection{Multi-Rate Controller with Interlacing (MRCI)}

The main goal of the interlacing technique is to reduce and uniformly distribute the amount of computation required at each fast sampling instance by a baseline controller $C(z)$. In order to reach it, $C(z)$ may be decomposed (in parallel or in serial decomposition), resulting in $m$ slow dynamics $C_{i}^{s}(z),(i=1, \ldots, m)$, and $n$ fast dynamics $C_{j}^{f}(z),(j=1, \ldots, n)$. The main idea is that the slow modes $C_{i}^{s}(z)$ will be implemented at an $m$ times slower rate without losing performance. Therefore, a multi-rate control approach with multi-rate ratio $m$ is needed. The conversion of the slow dynamics $C_{i}^{s}(z)$ to the slow rate $C_{i}^{s}\left(z^{m}\right)$ is carried out following this expression [8]

$$
C_{i}^{s}\left(z^{m}\right)=C_{i}\left(z^{m} I-A_{i}^{m}\right)^{-1}\left(\sum_{j=0}^{m-1} A_{i}^{m-1-j} B_{i}\right)+D_{i}
$$


where $\sum_{i}=\left\{A_{i}, B_{i}, C_{i}, D_{i}\right\}$ is a state-space realization for the slow mode $C_{i}^{s}(z)$.

Then, the set of slow modes is interlaced, that is, each $C_{i}^{s}\left(z^{m}\right)$ must be updated at instant $k=m j+i-1,(j \in \mathbb{N})$, instead of all at the same sampling instance. This fact implies a computation saving $C S$ given in $\%$ by

$$
C S=\frac{m-1}{m+n} \cdot 100 \%
$$

In order to do this interlacing operation (see in Figure 4), a down-sampling operator $(\downarrow m)$ is required at the input of each $C_{i}^{s}\left(z^{m}\right)$ to pick up the error signal $e_{k}=r_{k}-\hat{y}_{k}$ at every $m$ instances of the fast rate, and later, the consequent signal is delayed $z^{-(i-1)}$. Finally, a $\mathrm{ZOH}$ (at period $T$ ) is included at the output of each $C_{i}^{s}\left(z^{m}\right)$ to convert the slow-rate control signals to fast-rate ones. Every fast-rate control signal is summed along with the disturbance estimate $\hat{d}_{k}$ in order to get the control action $\hat{u}_{k}$. Following this operation mode for the next $\hat{y}_{k+l}$ and $\hat{d}_{k+l},(l=1, \ldots, h)$, the set of future control actions $\left\{\hat{u}_{k+1}, \ldots, \hat{u}_{k+h}\right\}$ can be obtained.

\subsubsection{Event-Triggered (ET) condition at the controller}

As a result of the estimation and design steps, the control signal $\left\{\hat{u}_{k}, \hat{u}_{k+1}, \ldots, \hat{u}_{k+h}\right\}$ is generated when $\beta_{k}=1$ (i.e. when a measurement is received by the control side from the sensor device).

Let $\gamma_{k} \in\{0,1\}$ denote the scheduling variable for the control signal in such a way that $\gamma_{k}=1$ denotes transmission of the control signal, and $\gamma_{k}=0$ otherwise. The first value $\hat{u}_{k}$ of the last sent control signal is stored in $\overline{\hat{u}}_{k}$ :

$$
\overline{\hat{u}}_{k}=\gamma_{k} \hat{u}_{k}+\left(1-\gamma_{k}\right) \overline{\hat{u}}_{k-1}
$$

The control signal is transmitted to the plant side using the following Mixed Triggered Mechanism (MTM):

$$
\left\|\overline{\hat{u}}_{k-1}-\hat{u}_{k}\right\|^{2}>\sigma_{c}\left\|\hat{u}_{k}\right\|^{2}+\delta_{c}
$$

where $\sigma_{c}$ and $\delta_{c}$ are positive constants.

Note that the feedback loop is only closed from plant side to control side, and back to plant side, when the conditions (6) (either (7) or (8)) and (22) hold (and hence, $\beta_{k}=\gamma_{k}=1$ ). If one of them fails (that is, $\beta_{k}=0$ or $\left.\gamma_{k}=0\right)$, then there is no update of the control signal, but the actuator can use the future control actions $\left\{\hat{u}_{k+1}, \ldots, \hat{u}_{k+h}\right\}$ previously sent. This modelbased control technique enables to retain a satisfactory control performance. 


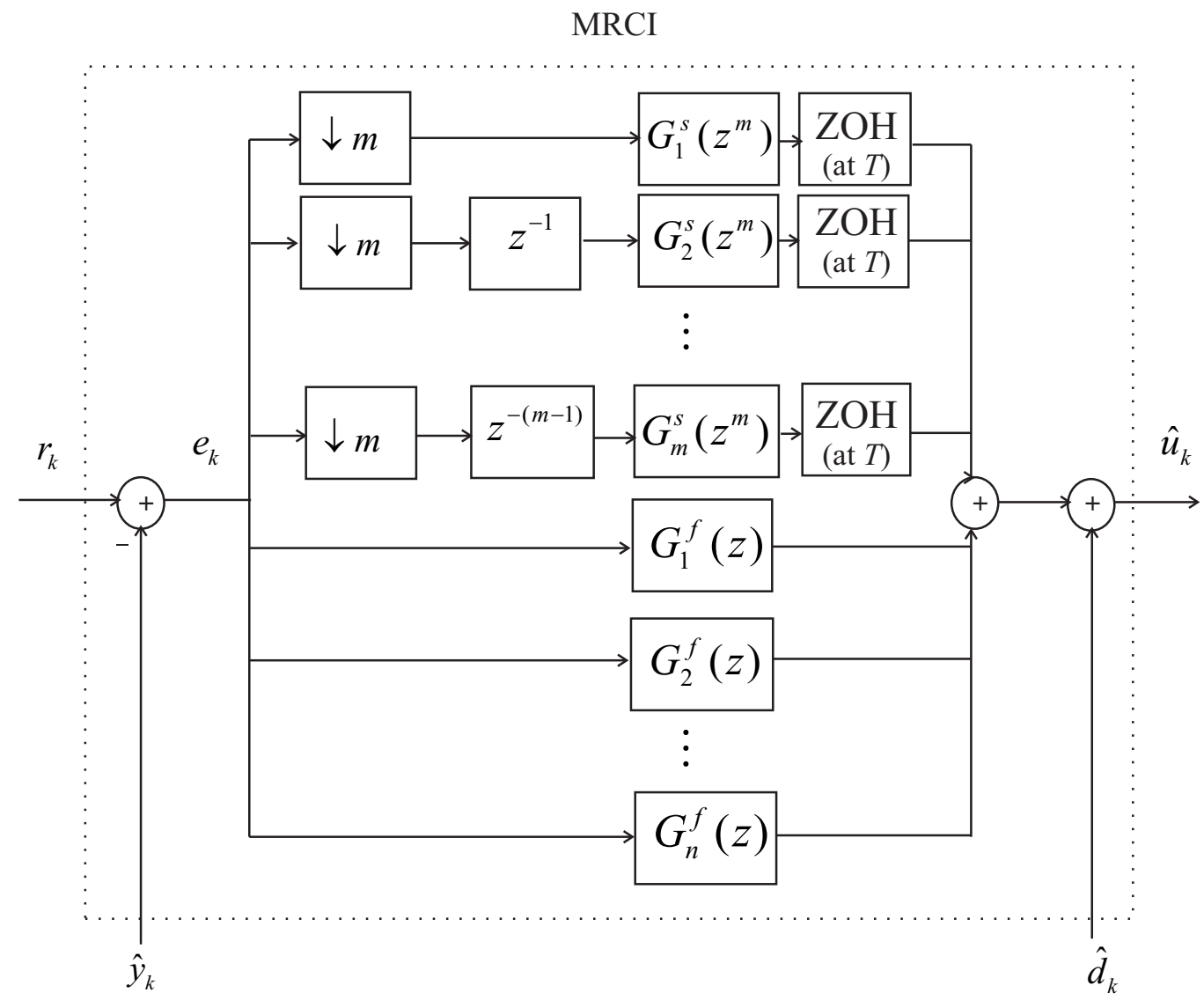

Figure 4: Structure of the Multi-Rate Controller with Interlacing

\section{Cost functions to analyze control performance and resource uti-} lization

In order to carry out an exhaustive evaluation of the control performance and resource utilization for the different sampling strategies, a set of cost functions can be used. Regarding control performance, similarly to [38], these indexes are considered:

- the Integral Squared Error (ISE), which provides a measure about how accurate the reference is followed in the Time-Triggered Sampling (TTS) strategy

$$
I S E=\sqrt{\sum_{k}\left(e_{k}^{\mathrm{TTS}}\right)^{2}}
$$


where $e_{k}^{\mathrm{TTS}}=r_{k}-y_{k}$.

- a kind of Integral Squared Error for the Periodic Event-Triggered Control solution $(I S E P)$, which compares the output obtained for the Event-Triggered Sampling (ETS) strategy with the one obtained for the TTS strategy (being used as a reference)

$$
I S E P=\sqrt{\sum_{k}\left|y_{k}^{\mathrm{ETS}}-y_{k}^{\mathrm{TTS}}\right|^{2}}
$$

- the cost function known as sampling efficiency $N_{E}$, which relates the performance of both sampling strategies

$$
N_{E}=\frac{I S E P}{I S E}
$$

ISE will be a constant value along the study, but ISEP will vary according to the ET conditions. The lower $N_{E}$ is, the better control performance will be for the PETC strategy.

To analyze the number of times in which the controller and the actuator are used in the PETC solution (i.e., the utilization of the controller $U_{c}$, and the utilization of the actuator $U_{a}$ ), the number of transmitted values from the sensor to the controller, $N_{s c}^{\mathrm{ETS}}$, and from the controller to the actuator, $N_{c a}^{\mathrm{ETS}}$, can be compared with the number of transmissions needed in the TTS approach, $N^{\mathrm{TTS}}$. In this way, the cost indexes $U_{c}$ and $U_{a}$ can be expressed as

$$
\begin{array}{r}
U_{c}=\frac{N_{s c}^{\mathrm{ETS}}}{N^{\mathrm{TTS}}} \\
U_{a}=\frac{N_{c a}^{\mathrm{ETS}}}{N^{\mathrm{TTS}}}
\end{array}
$$

Taking into account the computation saving reached by including the interlacing technique in (20), the amount of computation required by the controller, $C_{c}$, can be defined in $\%$ as follows

$$
C_{c}=U_{c}\left(1-\frac{m-1}{m+n}\right) \cdot 100 \%
$$

Finally, to summarize the different cost indexes, a Generalized Performance Index $(G P I)$ is introduced (as similarly defined in [38]):

$$
G P I=W_{1} U_{c}+W_{2} U_{a}+W_{3} N_{E}
$$


where $\sum_{i=1}^{3} W_{i}=1 . W_{i}$ are weights indicating the importance of the corresponding factor ( $W_{1}$ and $W_{2}$ are related to resource utilization, and $W_{3}$ is connected to control performance). The selection of the weight values $W_{i}$ in practical applications depends on the following factors:

- User requirements on $I S E$ and the number of corresponding events. The later depends also on the characteristics of used devices, e.g. CPU runtime properties, communication protocol, etc.

- Assignment of the control elements to automation nodes. For example, if the controller and the actuator are implemented in one node, then $W_{2}$ is set to 0 .

GPI indicates the trade-off between event rates and control performance. The less this index is, the better the overall performance will be. In this work, GPI will be mainly used to find optimal values for the thresholds included in the ET conditions (6) and (22).

\section{Simulation results via Truetime}

Here, a practical Hard Disk Drive (HDD) model with two large resonances is used to perform the simulation study. This HDD model is modified from the HDD Benchmark model [20]. The HDD model with resonances is described by

$$
G=\frac{7.153 \cdot 10^{6}}{s^{2}+502.7 s+1.011 \cdot 10^{6}}\left(\prod_{i=1}^{i=2} \frac{k_{v i} w_{i}^{2}}{s^{2}+2 \zeta_{i} w_{i} s+w_{i}^{2}}\right)
$$

where $k_{v i}(i=1,2)$ are the gains of resonances; $w_{i}(i=1,2)$ are the resonance frequencies; $\zeta_{i}(i=1,2)$ denotes the corresponding damping ratios. The plant model is discretized at period $T=20 \mu \mathrm{s}$. The parameters of the resonances are provided in Table 2 . These frequency resonances are attenuated by notch filters. Here the notch filters are designed as follows:

$$
N=\Pi_{i=1}^{2} \frac{1-2 \alpha_{i} \cos \left(w_{i} T\right) z^{-1}+\alpha_{i}^{2} z^{-2}}{1-2 \beta_{i} \cos \left(w_{i} T\right) z^{-1}+\beta_{i}^{2} z^{-2}}
$$

All the parameters are provided in Table 2. 
Table 2: Parameters in HDDs

\begin{tabular}{|l|l|l|l|l|l|}
\hline$i$ & $k_{i}$ & $w_{i}(\mathrm{~Hz})$ & $\zeta_{i}$ & $\alpha_{i}$ & $\beta_{i}$ \\
\hline 1 & 0.02 & 5000 & 0.03 & 0.965 & 0.975 \\
2 & 0.01 & 10000 & 0.01 & 0.3 & 0.3 \\
\hline
\end{tabular}

- The plant to be controlled includes the resonances and notch filters, which results in a $10^{\text {th }}$-order model that is considered to represent the actual HDD plant in a good extent in existing literature $[20,5]$. It was converted to the state space realization (1) in the simulation.

- The disturbance is defined as in (2) at period $T$ by means of

$$
\begin{aligned}
A_{d} & =\left[\begin{array}{cc}
1 & 0 \\
-2.8419 & 1
\end{array}\right] \\
B_{d} & =\left[\begin{array}{c}
0 \\
0.1508 \cdot 10^{-3}
\end{array}\right] \\
C_{d} & =\left[\begin{array}{ll}
0 & 1 \cdot 10^{5}
\end{array}\right]
\end{aligned}
$$

- A digital controller is designed at period $T$

$$
C_{D}(z)=\frac{1.0882(z-0.9813)(z-0.9681)(z+1)}{(z-1)(z-0.8496)(z-0.6137)}
$$

where two slow modes (poles in $z=0.8496$ and $z=1$ ) and one fast mode (pole in $z=0.6137$ ) are detected. Then, considering (19), the controller can be decomposed from the multi-rate with interlacing perspective $(m=2)$ for parallel implementation as

$$
\begin{aligned}
C_{1}^{s}\left(z^{2}\right) & =\frac{0.04484}{z^{2}-1} \\
C_{2}^{s}\left(z^{2}\right) & =\frac{-1.6365}{z^{2}-0.7218} \\
C_{1}^{f}(z) & =\frac{1.0882 z+1.84}{z-0.6137}
\end{aligned}
$$

implying, according to (20), a computation saving $C S=33 \%$.

- The Non-Uniform Multi-Rate Estimator is designed considering the augmented state resulting from the $10^{\text {th }}$-order plant model (from (30)(31)) and from the disturbance (32), when the disturbance dynamics 
are known; otherwise, it is designed taking into account only the plant model.

- The Disturbance Observer is designed through solving the $H_{\infty}$ optimization problem (17).

- The constant values in the Event-Triggered (ET) conditions (6) and (22) will be respectively $\delta_{s}=\sigma_{s}=100 \cdot 10^{-7}$ and $\delta_{c}=\sigma_{c}=1.32 \cdot 10^{-2}$. These values are obtained as the optimal ones when giving preference to resource saving in (29), that is, choosing the weights $W_{1}, W_{2}$ greater than $W_{3}$. In this case, the selection is $W_{1}=W_{2}=0.45, W_{3}=0.1$. Evaluating the ET conditions for different thresholds (choosing, for the sake of simplicity, $\delta_{s}=\sigma_{s}$ and $\delta_{c}=\sigma_{c}$ ) the consequent values for the Generalized Performance Index GPI are obtained, generating the surface depicted in Figure 5. The optimal values for the thresholds are achieved from the lowest GPI value. Finally, the Time-Triggered (TT) conditions $(7)$ or $(8)$ at the sensor are not required in this example.

5.1. Case 1: Time-Triggered Sampling (TTS) scenario with no noise, no disturbance, no Non-Uniform Multi-Rate Estimator (NUMRE)

The results obtained in this case are illustrated in Figure 6, yielding the Integral Squared Error $I S E=3.75$ and the number of transmissions carried out in the TTS approach $N^{\mathrm{TTS}}=464$. The top subplot shows the desired system response. The middle subplot presents the consequent control signal. The bottom subplot indicates the utilization of the controller and actuator by including a bar at each sampling instance when the devices are used. As a TTS case, the utilization for controller and actuator is $U_{c}=U_{a}=1$ (since, as expected, every measurement/control action is sent). Note that, due to the interlacing technique, the total amount of computation will be $C_{c}=66 \%$.

5.2. Case 2: Periodic Event-Triggered Control (PETC) scenario with no noise, no disturbance, no Non-Uniform Multi-Rate Estimator (NUMRE)

This case is shown in Figure 7, where:

- the number of transmitted values from the sensor to the controller is $N_{s c}^{\mathrm{ETS}}=250$ and hence the utilization of the controller is $U_{c}=0.54$, which implies reducing $46 \%$ the amount of packets transmitted in comparison with the TTS case. In addition, the total amount of computation will be $C_{c}=35 \%$, which approximately represents a 31 percentage point decrease in computation with regard to the TTS strategy. 


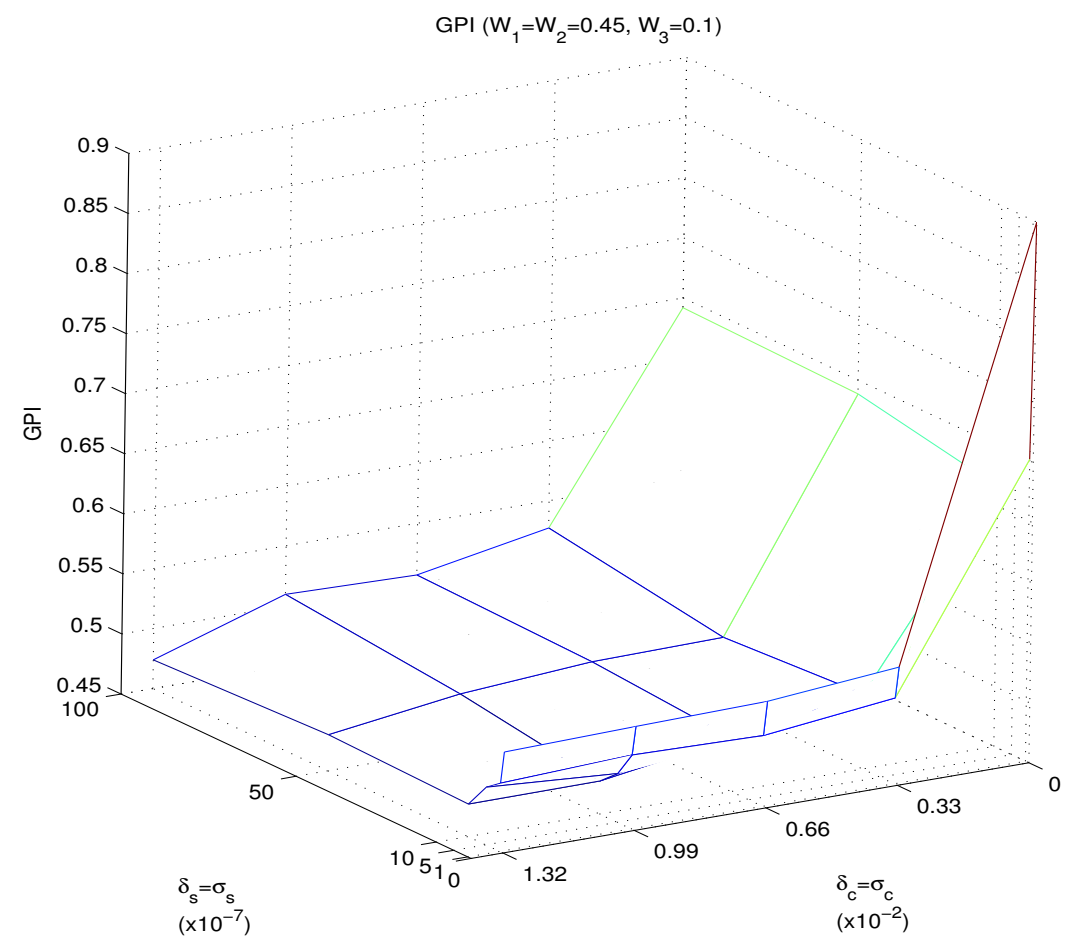

Figure 5: GPI results ( $W_{i}$ for resource saving) 
TTS
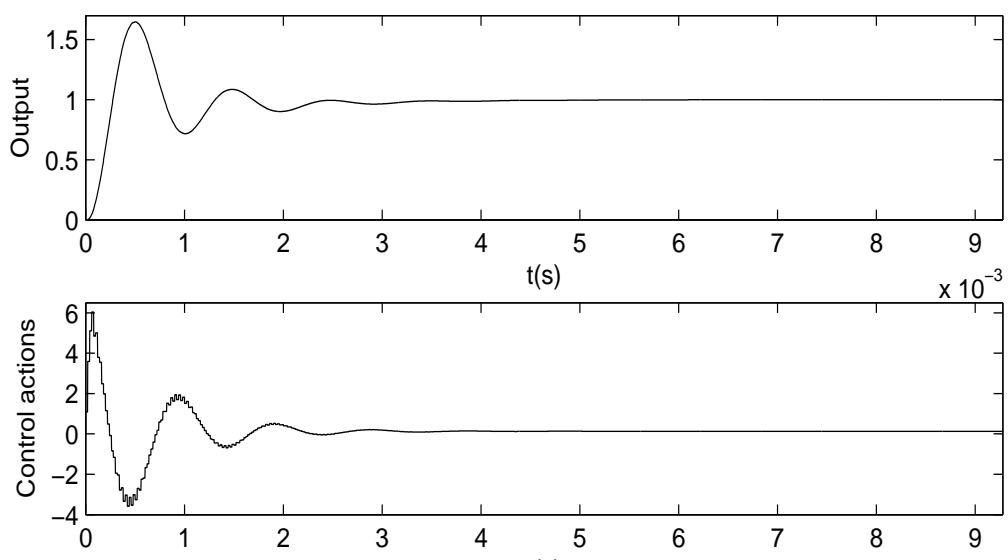

$\mathrm{t}(\mathrm{s})$

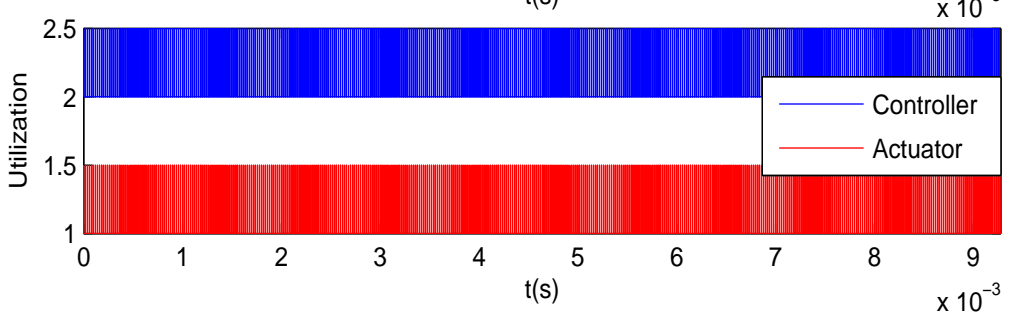

Figure 6: TTS results (no noise, no disturbance, no estimator) 

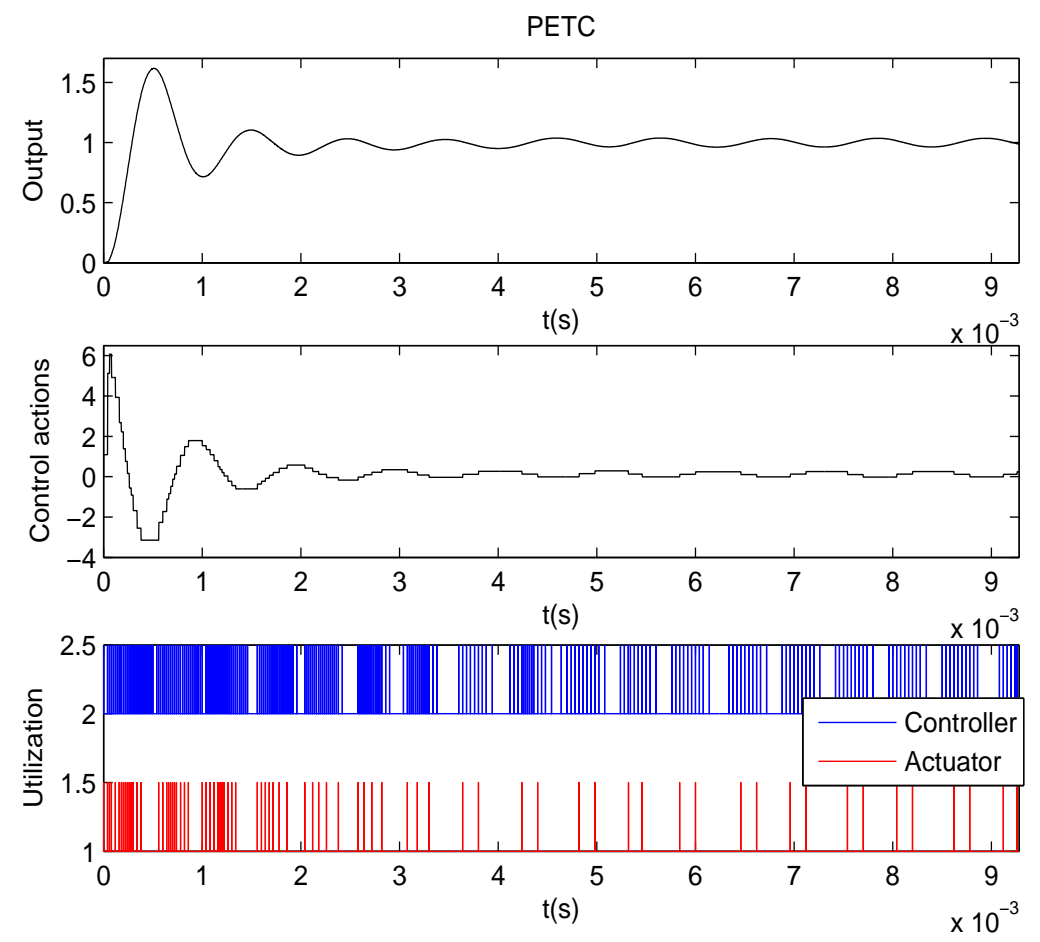

Figure 7: PETC results (no noise, no disturbance, no estimator)

- the number of transmitted values from the controller to the actuator is $N_{c a}^{\mathrm{ETS}}=78$ and hence the utilization of the actuator is $U_{a}=0.16$, which leads to reduce $84 \%$ the data transferred from controller to actuator.

- the Integral Squared Error for the Periodic Event-Triggered Control solution is $I S E P=0.54$ and hence the sampling efficiency becomes $N_{E}=0.14$, representing a considerable worsening of control performance $(14 \%)$, which is mainly noticed at the steady-state response.

\subsection{Case 3: Periodic Event-Triggered Control (PETC) scenario with no} noise, no disturbance, but Non-Uniform Multi-Rate Estimator (NUMRE)

In this case, the NUMRE has been implemented in the PETC scenario, reaching the expected results (see in Figure 8), that is, the control system is able to achieve the desired performance (that obtained for the TTS case, shown in Figure 6) despite clearly reducing resource usage. Now: 

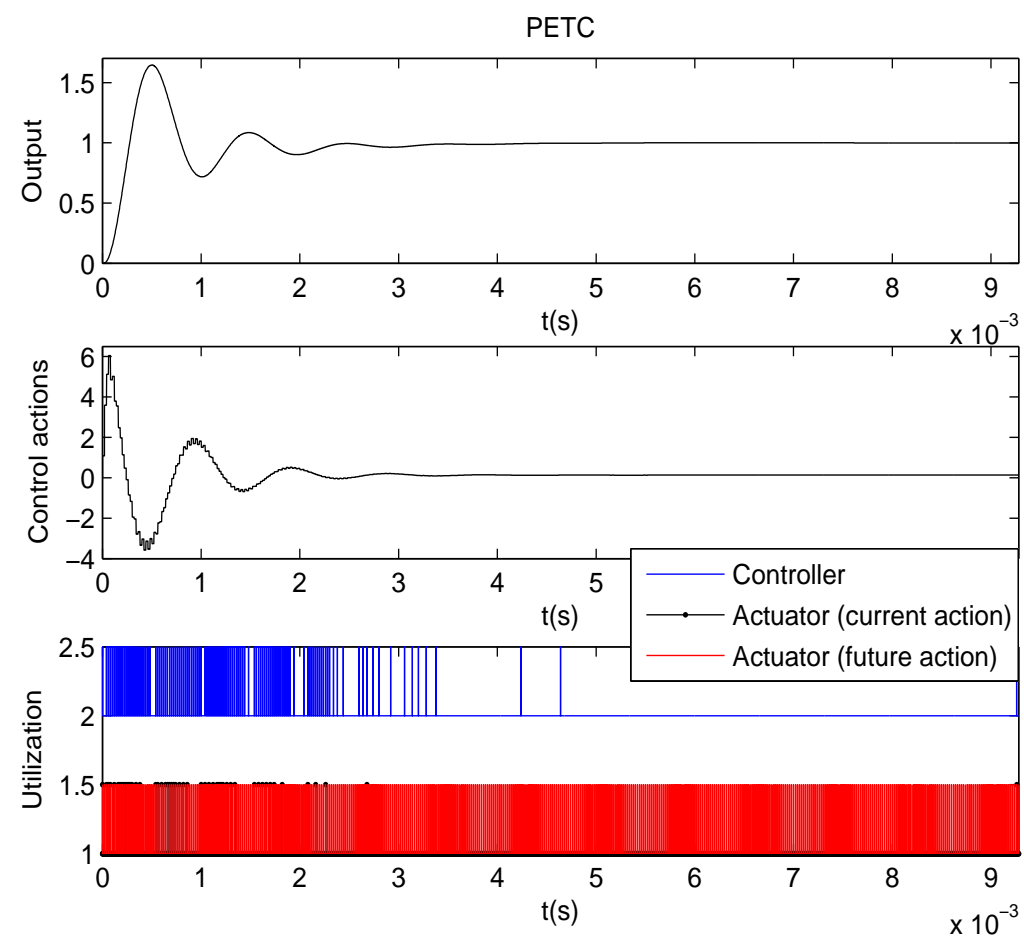

Figure 8: PETC results (NUMRE, no noise, no disturbance)

- $N_{s c}^{\mathrm{ETS}}=120$, and hence, $U_{c}=0.26$ and $C_{c}=17 \% . C_{c}$ represents a 18 percentage point decrease in computation with respect to that obtained in Case 2, and 49 regarding the TTS case.

- $N_{c a}^{\mathrm{ETS}}=50$, and hence $U_{a} \approx 0.1$, which implies reducing $36 \%$ the amount of transmitted packets in comparison with Case 2, and around 90\% regarding the TTS case.

Note that, when considering the NUMRE, the actuator is always applying a control action, that is, when the Event-Triggered condition at the control side is triggered, the actuator applies the current control action (marked by a black point at the bottom subplot in Figure 8); otherwise, a future control action is applied. 
5.4. Case 4: Periodic Event-Triggered Control (PETC) scenario with noise, disturbance (knowing its dynamic model), and Non-Uniform Multi-Rate Estimator (NUMRE)

In this case (shown in Figure 9):

- $N_{s c}^{\mathrm{ETS}}=203\left(U_{c}=0.44, C_{c}=29 \%\right)$ and $N_{c a}^{\mathrm{ETS}}=77\left(U_{a}=0.15\right)$ and hence, in comparison with the TTS strategy, the transferred data have been respectively reduced around $56 \%$ and $85 \%$, resulting a 37 percentage point decrease in computation. With respect to Case 2, these values represent respectively $10 \%$ and $1 \%$ of additional reduction of sensor-tocontroller and controller-to-actuator transmitted packets, and 17 percentage point decrease in computation.

- However, as shown in Figure 9, the behavior of the estimated output is worsened at the steady-state response in comparison with that obtained in the TTS strategy (the sampling efficiency becomes $N_{E}=9 \%$ ). Contrarily, $N_{E}$ represents a 5 percentage point decrease compared to the Case 2.

Thus, despite including noise and disturbance in the PETC scenario, the consideration of the NUMRE continues being beneficial both for resource saving and for control performance. Although, as expected, the fact of including noise and disturbance has increased the devices' utilization with respect to those obtained in Case 3. Mainly, the utilization of the controller $U_{c}$ has experimented around a 20 percentage point increase.

The performance worsening with regard to the Time-Triggered Sampling case can be explained observing the control signal, which is negatively affected by a not accurate estimation of the disturbance (as shown at the top subplot in Figure 10). This is due to the consideration of wide thresholds in the Event-Triggered conditions (remember that they were chosen to give priority to resource saving in Figure 5), which implies higher values for the time-varying multiplicity $N$, mainly at the steady-state response (as depicted in the middle plot in Figure 10), and then, worse estimations. Note that $N=0$ at the bottom subplot in Figure 10 means no event is triggered at the sensor device, which is quite probable in this case as a consequence of the chosen thresholds. 


\section{PETC}

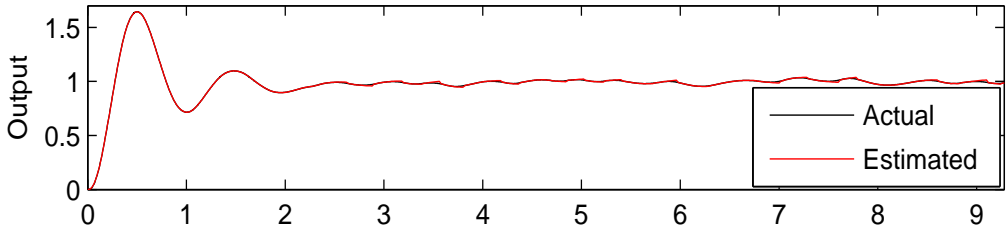

$t(s)$

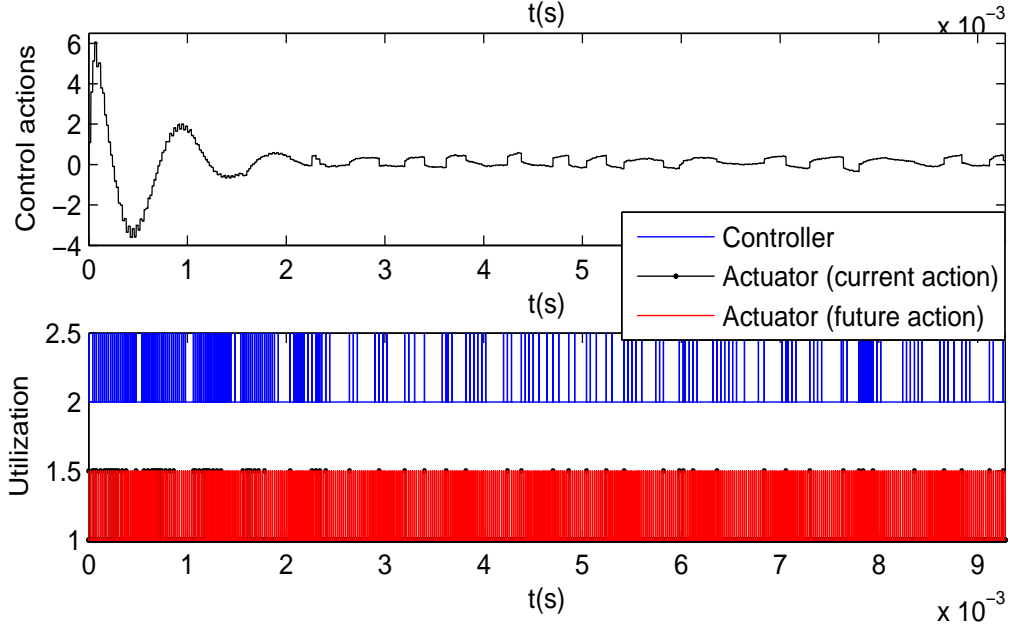

Figure 9: PETC results (NUMRE, noise, disturbance) 

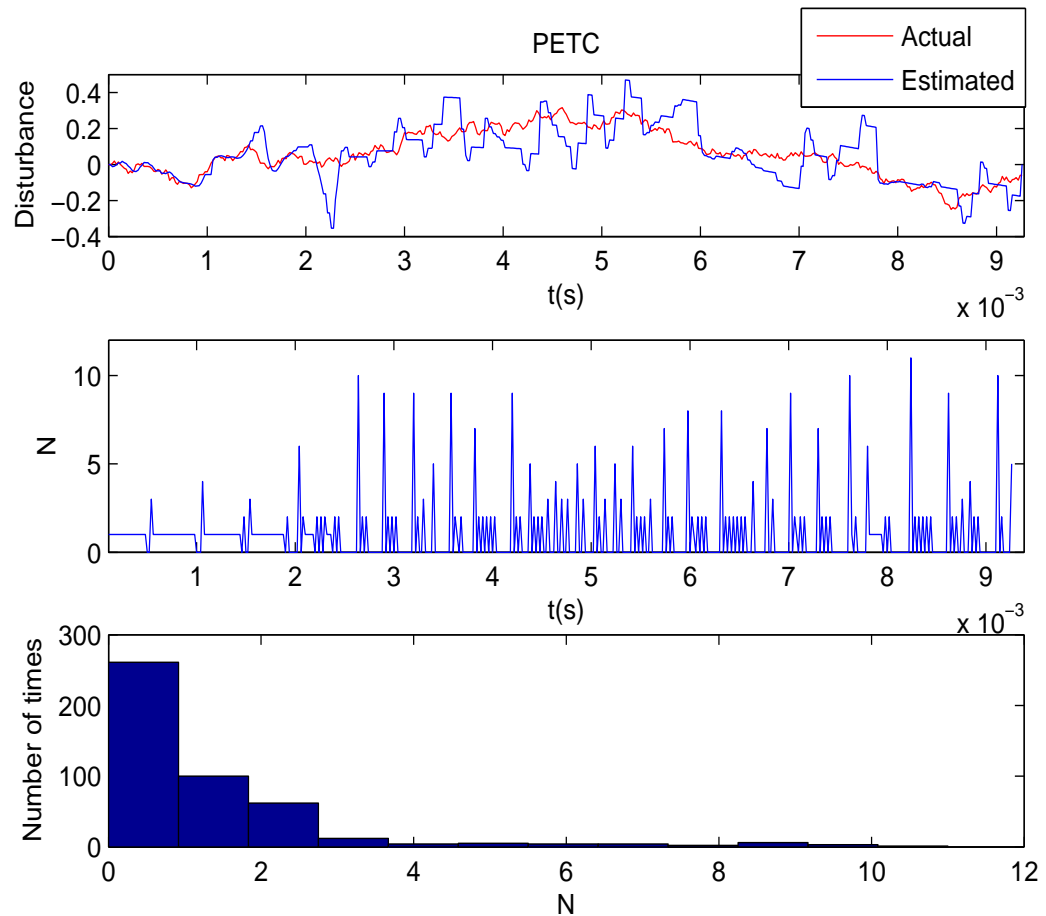

Figure 10: PETC results: disturbance signal and multiplicity $N$ 
5.5. Case 5: Periodic Event-Triggered Control (PETC) scenario (narrower thresholds) with noise, disturbance (knowing its dynamic model), and Non-Uniform Multi-Rate Estimator (NUMRE)

In this case (Figure 11), narrower thresholds has been chosen (concretely, $\delta_{s}=\sigma_{s}=1 \cdot 10^{-7}$ and $\left.\delta_{c}=\sigma_{c}=1 \cdot 10^{-4}\right)$. As expected, compared to the previous case, now the number of transmitted packets is increased $\left(N_{s c}^{\mathrm{ETS}}=338\right.$, and hence $U_{c}=0.73, C_{c}=48 \%$; and $N_{c a}^{\mathrm{ETS}}=231$ ). But compared to the TimeTriggered Sampling scenario, these results still represent a 18 percentage point decrease in computation, and around $27 \%$ and $52 \%$ respectively of reduction in sensor-to-controller and controller-to-actuator transferred packets. These figures are achieved while practically maintaining the desired performance $\left(N_{E}=2 \%\right)$. Therefore, this case illustrates the trade-off between resource saving and control performance.

Now, as depicted in Figure 12, the disturbance is accurately estimated, since lower values for $N$ are achieved (due to the consideration of narrower thresholds in the Event-Triggered conditions). As expected, the likelihood of not triggering an event $(N=0)$ is lower than in the previous case.

5.6. Case 6: Periodic Event-Triggered Control (PETC) scenario (narrower thresholds) with noise, disturbance (no dynamic model), Non-Uniform Multi-Rate Estimator (NUMRE), and Disturbance Observer (DOB)

In this last study, as the dynamic model of the disturbance is not known, a DOB is added. Figure 13 shows the results. Compared to the previous case, a similar computation saving is achieved $\left(N_{s c}^{E T S}=360\right.$, and hence $U_{c}=0.78$, $\left.C_{c}=53 \%\right)$ but sending around $15 \%$ more packets from controller to actuator $\left(N_{c a}^{E T S}=315\right)$. Although the control signal presents some oscillations in the steady-state response (because $N$ tends to be higher, and then the disturbance estimation is not so accurate), very similar performance to that achieved in the previous case is obtained $(N E=1 \%)$. In summary, despite relaxing the knowledge of the disturbance model, similar resources are used, leading to similar system behavior.

\section{Conclusions}

The main benefit of using Event-Triggered Sampling strategies together with multi-rate estimation and control techniques (via Non-Uniform MultiRate Estimator and Multi-Rate Controller with Interlacing) is to achieve resource saving while maintaining control performance at approximately the 

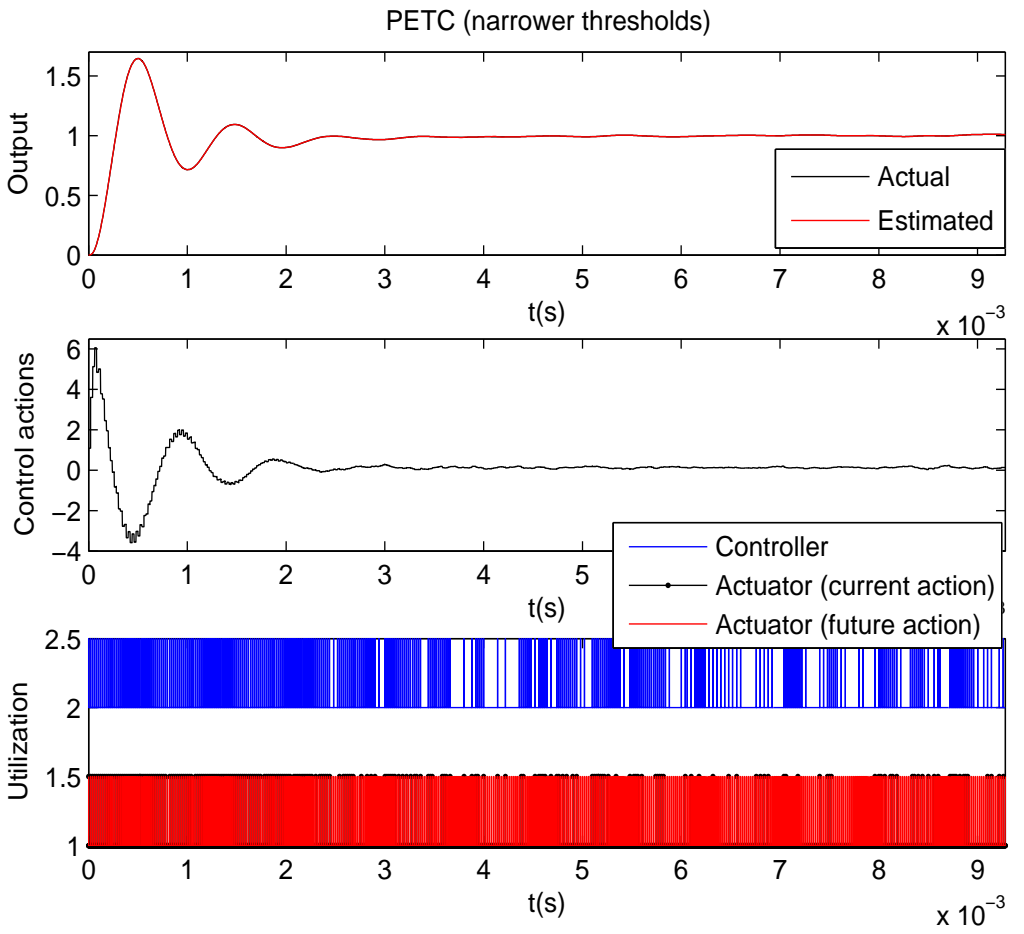

Figure 11: PETC results (narrower thresholds, NUMRE, noise, disturbance) 

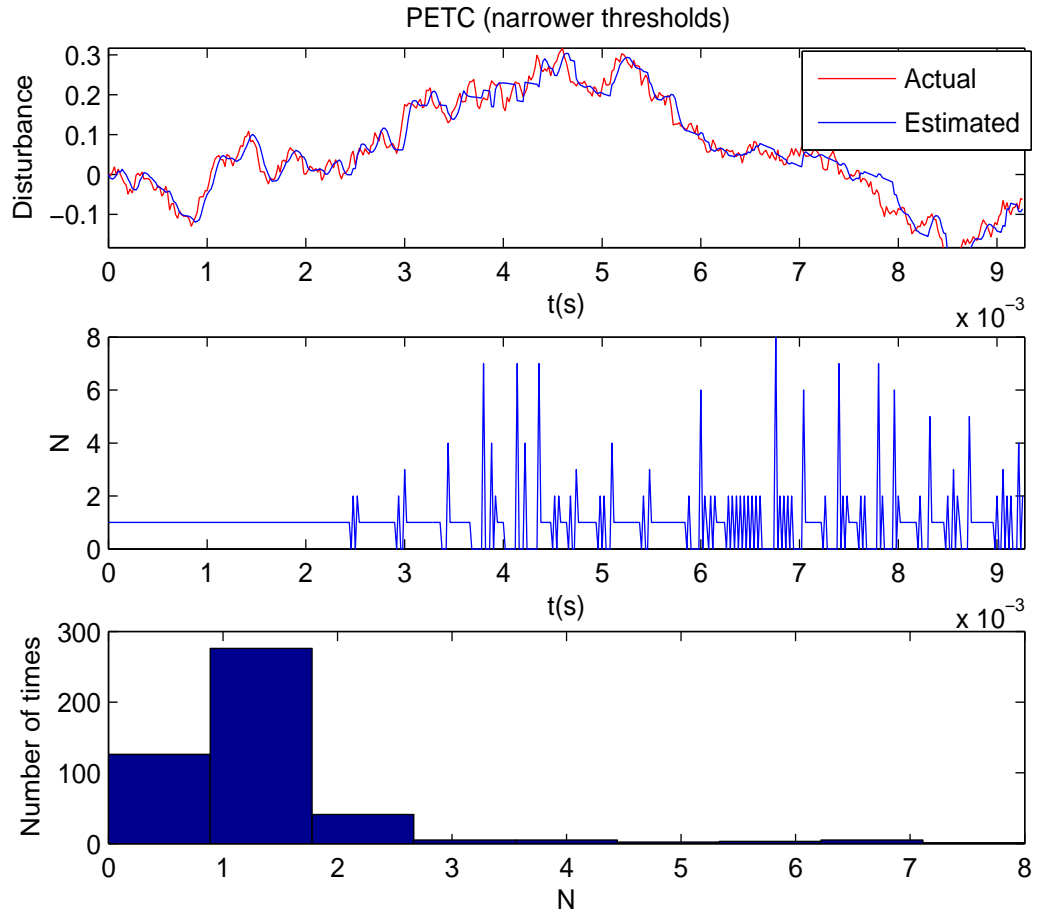

Figure 12: PETC results (narrower thresholds): disturbance signal and multiplicity $N$ 

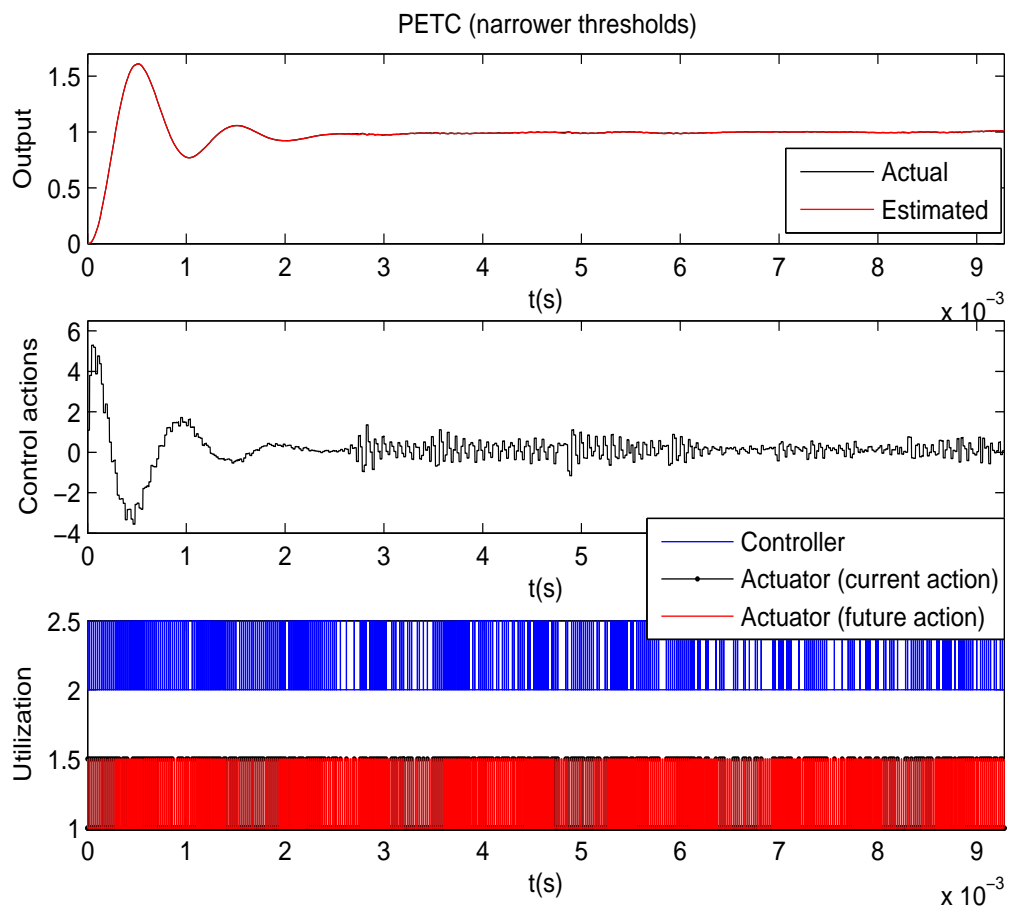

Figure 13: PETC results (narrower thresholds, NUMRE, DOB, noise, disturbance with no model) 
same level as the Time-Triggered Sampling solution. As analyzed, some trade-off between the performance and the saving can be reached by conveniently tuning the thresholds of the Event-Triggered conditions. In ideal conditions (with no noise and no disturbance), significant reduction of transmitted packets and computation is attained while the desired performance is maintained. When the noise and disturbance are added, the average reduction is considerable but some performance compromise is observed. To reach the desired performance in presence of disturbance and noise, the thresholds in the Event-Triggered conditions have to be narrower while lessening the average resource saving. These results can be obtained by adding a Disturbance Observer as an alternative when the dynamic model of the disturbance is not known.

\section{Acknowledgements}

This research work has been developed as a result of a mobility stay funded by the Spain Visiting Fulbright Scholar Programme of the Fulbright Commission and the Spanish Ministry of Education under "Programa Estatal de Promoción del Talento y su Empleabilidad en I+D+i, Subprograma Estatal de Movilidad, del Plan Estatal de Investigación Científica y Técnica y de Innovación 2013-2016".

In addition, the work is funded by European Commission as part of Project H2020-SEC-2016-2017 - Topic: SEC-20-BES-2016 - Id: 740736 - "C2 Advanced Multi-domain Environment and Live Observation Technologies" (CAMELOT). Part WP5 supported by Tekever ASDS, Thales Research and Technology, Viasat Antenna Systems, Universitat Politècnica de València, Fundação da Faculdade de Ciências da Universidade de Lisboa, Ministério

da Defensa Nacional - Marinha Portuguesa, Ministério da Administração Interna Guarda Nacional Republicana.

\section{References}

[1] J. Bae, W. Zhang, M. Tomizuka, Network-based rehabilitation system for improved mobility and tele-rehabilitation, IEEE Trans. Control Syst. Technol. 21 (5) (2013) 1980-1987.

[2] D. P. Borgers, W. M. H. Heemels, Event-separation properties of eventtriggered control systems, IEEE Trans. Autom. Control 59 (10) (2014) 2644-2656. 
[3] V. Casanova, J. Alcaina, J. Salt, R. Piza, A. Cuenca, Control of the rotary inverted pendulum through threshold-based communication, ISA Trans. 62 (2016) 357-366.

[4] A. Cervin, D. Henriksson, B. Lincoln, J. Eker, K.-E. Arzen, How does control timing affect performance? Analysis and simulation of timing using Jitterbug and TrueTime, IEEE Control Syst. 23 (3) (2003) 16-30.

[5] X. Chen, M. Tomizuka, A minimum parameter adaptive approach for rejecting multiple narrow-band disturbances with application to hard disk drives, IEEE Trans. Control Syst. Technol. 20 (2) (2012) 408-415.

[6] A. Cuenca, P. Garcia, P. Albertos, J. Salt, A non-uniform predictorobserver for a networked control system, Int. J. Control, Autom. Syst. 9 (6) (2011) 1194-1202.

[7] A. Cuenca, J. Alcaina, J. Salt, V. Casanova, R. Piza, A packet-based dual-rate PID control strategy for a slow-rate sensing networked control system, ISA Trans. 76 (2018) 155-166.

[8] J. Ding, F. Marcassa, S.-C. Wu, M. Tomizuka, Multirate control for computation saving, IEEE Trans. Control Syst. Technol. 14 (1) (2006) 165-169.

[9] L. Ding, Q.-L. Han, G. Guo, Network-based leader-following consensus for distributed multi-agent systems, Automatica 49 (7) (2013) 22812286 .

[10] L. Ding, Q.-L. Han, X. Ge, X.-M. Zhang, An overview of recent advances in event-triggered consensus of multiagent systems, IEEE Trans. Cybern. 48 (4) (2018) 1110-1123.

[11] E. Garcia, P. J. Antsaklis, Model-based event-triggered control for systems with quantization and time-varying network delays, IEEE Trans. Autom. Control 58 (2) (2013) 422-434.

[12] X. Ge, Q.-L. Han, Distributed event-triggered $H_{\infty}$ filtering over sensor networks with communication delays, Inf. Sci. 291 (2015) 128-142. 
[13] X. Ge, Q.-L. Han, Z. Wang, A dynamic event-triggered transmission scheme for distributed set-membership estimation over wireless sensor networks, IEEE Trans. Cybern. (2017). In Press: 10.1109/TCYB.2017.2769722.

[14] X. Ge, F. Yang, Q.-L. Han, Distributed networked control systems: A brief overview, Inf. Sci. 380 (2017) 117-131.

[15] X. Ge, Q.-L. Han, and Z. Wang. A threshold-parameter-dependent approach to designing distributed event-triggered $H_{\infty}$ consensus filters over sensor networks. IEEE Trans. Cybern. (2018). In Press: 10.1109/TCYB.2017.2789296.

[16] W. Heemels, M. Donkers, Model-based periodic event-triggered control for linear systems, Automatica 49 (3) (2013) 698-711.

[17] W. Heemels, R. Gorter, A. van Zijl, P. Van den Bosch, S. Weiland, W. Hendrix, M. Vonder, Asynchronous measurement and control: a case study on motor synchronization, Control Eng. Prac. 7 (12) (1999) 14671482 .

[18] W. Heemels, M. Donkers, A. R. Teel, Periodic event-triggered control for linear systems, IEEE Trans. Autom. Control 58 (4) (2013) 847-861.

[19] S. Hu, D. Yue, Event-based $H_{\infty}$ filtering for networked system with communication delay, Signal Process. 92 (9) (2012) 2029-2039.

[20] IEEJ, IEEJ Tech. Comm. for Novel Nanoscale Servo Control, NSS benchmark problem of Hard Disk Drive Syst., http://mizugaki.iis.utokyo.ac.jp/nss/.

[21] P. Khargonekar, K. Poolla, A. Tannenbaum, Robust control of linear time-invariant plants using periodic compensation, IEEE Trans. Autom. Control 30 (11) (1985) 1088-1096.

[22] K. Kong, J. Bae, M. Tomizuka, Control of rotary series elastic actuator for ideal force-mode actuation in humanrobot interaction applications, IEEE/ASME Trans. Mechatron. 14 (1) (2009) 105-118.

[23] C. Liu, M. Tomizuka, Designing the robot behavior for safe human-robot interactions, in: Trends in Control and Decision-Making for HumanRobot Collab. Syst., 2017, pp. 241-270. 
[24] Q. Liu, Z. Wang, X. He, D. Zhou, Event-based recursive distributed filtering over wireless sensor networks, IEEE Trans. Autom. Control 60 (9) (2015) 2470-2475.

[25] J. Lunze, D. Lehmann, A state-feedback approach to event-based control, Automatica 46 (1) (2010) 211-215.

[26] R. McCann, A. T. Le, Lebesgue sampling with a Kalman filter in wireless sensors for smart appliance networks, in: Ind. Appl. Soc. Annu. Meet., 2008, pp. 1-5.

[27] V. H. Nguyen, Y. S. Suh, Networked estimation for event-based sampling systems with packet dropouts, Sensors 9 (4) (2009) 3078-3089.

[28] C. Peng, M.-R. Fei, E. Tian, Y.-P. Guan, On hold or drop out-of-order packets in networked control systems, Inf. Sci. 268 (2014) 436-446.

[29] C. Peng, D. Yue, M.-R. Fei, A higher energy-efficient sampling scheme for networked control systems over IEEE 802.15.4 wireless networks, IEEE Trans. Ind. Informat. 12 (5) (2016) 1766-1774.

[30] J. Salt, M. Tomizuka, Hard disk drive control by model based dualrate controller. Computation saving by interlacing, Mechatronics 24 (6) (2014) 691-700.

[31] J. Sanchez, M. A. Guarnes, S. Dormido, On the application of different event-based sampling strategies to the control of a simple industrial process, Sensors 9 (9) (2009) 6795-6818.

[32] J. Sijs, M. Lazar, On event based state estimation, in: Int. Workshop Hybrid Syst.: Comput. and Control, 2009, pp. 336-350.

[33] D. Simon, Optimal state estimation: Kalman, $H_{\infty}$, and nonlinear approaches, John Wiley \& Sons, 2006.

[34] B. Sinopoli, L. Schenato, M. Franceschetti, K. Poolla, M. I. Jordan, S. S. Sastry, Kalman filtering with intermittent observations, IEEE Trans. Autom. Control 49 (9) (2004) 1453-1464.

[35] A. Smyth, M. Wu, Multi-rate Kalman filtering for the data fusion of displacement and acceleration response measurements in dynamic system monitoring, Mech. Syst. Signal Process. 21 (2) (2007) 706-723. 
[36] P. Tabuada, Event-triggered real-time scheduling of stabilizing control tasks, IEEE Trans. Autom. Control 52 (9) (2007) 1680-1685.

[37] S. Trimpe, R. D'Andrea, Event-based state estimation with variancebased triggering, IEEE Trans. Autom. Control 59 (12) (2014) 3266-3281.

[38] V. Vasyutynskyy, K. Kabitzsch, Towards comparison of deadband sampling types, in: Int. Symp. Ind. Electron., 2007, pp. 2899-2904.

[39] D. Wang, J. Wang, W. Wang, Output feedback control of networked control systems with packet dropouts in both channels, Inf. Sci. 221 (2013) 544-554.

[40] J. Wang, X.-M. Zhang, Q.-L. Han, Event-triggered generalized dissipativity filtering for neural networks with time-varying delays, IEEE Trans. Neural Netw. Learn. Syst. 27 (1) (2016) 77-88.

[41] Y. Xia, W. Xie, B. Liu, X. Wang, Data-driven predictive control for networked control systems, Inf. Sci. 235 (2013) 45-54.

[42] W. Zhang, M. S. Branicky, S. M. Phillips, Stability of networked control systems, IEEE Control Syst. 21 (1) (2001) 84-99.

[43] X.-M. Zhang, Q.-L. Han, Event-triggered dynamic output feedback control for networked control systems, IET Control Theory Appl. 8 (4) (2014) 226-234.

[44] X.-M. Zhang, Q.-L. Han, Event-triggered $H_{\infty}$ control for a class of nonlinear networked control systems using novel integral inequalities, Int. J. Robust Nonlin. Control 27 (4) (2017) 679-700.

[45] X.-M. Zhang, Q.-L. Han, B.-L. Zhang, An overview and deep investigation on sampled-data-based event-triggered control and filtering for networked systems, IEEE Trans. Ind. Informat. 13 (1) (2017) 4-16.

[46] Y.-B. Zhao, G.-P. Liu, D. Rees, Actively compensating for data packet disorder in networked control systems, IEEE Trans. Circuits Syst. II: Express Briefs 57 (11) (2010) 913-917.

[47] M. Zheng, X. Chen, M. Tomizuka, Extended state observer with phase compensation to estimate and suppress high-frequency disturbances, in: American Control Conf., 2016, pp. 3521-3526. 
[48] M. Zheng, L. Sun, M. Tomizuka, Multi-rate observer based sliding mode control with frequency shaping for vibration suppression beyond Nyquist frequency, IFAC-PapersOnLine 49 (21) (2016) 13-18.

[49] M. Zheng, S. Zhou, M. Tomizuka, A design methodology for disturbance observer with application to precision motion control: An $H_{\infty}$ based approach, in: American Control Conf., 2017, pp. 3524-3529. 